\title{
A STRATEGY TO QUALIFY THE PERFORMANCE OF RADIOGRAPHIC MONITORS
}

\author{
D. Gutierrez, P. Monnin, J.-F. Valley and F. R. Verdun*
}

University Institute for Applied Radiophysics, Grand-Pré 1, CH-1007 Lausanne, Switzerland

The purpose of this work was to compare standard desktop display systems with dedicated medical display systems. The set of image tests proposed by the American Association of Physicists in Medicine (AAPM TG18) was used to assess a Philips 107S desktop display system and a Siemens medical display. Three observers performed the subjective assessment, in a non-concerted manner. The objective assessment was performed using a CCD camera according to the AAPM TG18 procedure. The results clearly demonstrate the inadequacy of standard desktop display systems in the framework of diagnostic radiology. Moreover, a good correlation between the subjective and objective assessment methods was obtained.

\section{INTRODUCTION}

The weakest link in conventional radiology is the film processing. To assure the stability of image quality produced over time, a quality assurance programme requiring frequent control of film processing is mandatory. In mammography screening programmes, for example, the stability of film processing is required at least once a day ${ }^{(1,2)}$. The transition from conventional to digital poses another type of problem for us, since the film printers remove this weakness, because these processors have self-control devices, which ensure the production of films of constant quality.

However, one should not ignore that a weakness still exists in the radiological chain. The new weakest link is the monitor on which radiologists read their images. In this context, acceptance test and stability tests should be applied to diagnostic monitors to assure that no diagnostic information is lost over time.

To help radiologists, medical physicists and radiographers control the adequacy of their monitors the American Association of Physicists in Medicine (AAPM) has proposed a set of test images allowing both subjective and objective assessments of monitors $^{(3)}$. The goal of the work is to apply several of these test images in order to evaluate their sensitivity and to establish a policy concerning the quality control of the monitors in our institution by following well-known image quality parameters such as resolution and noise ${ }^{(4)}$.

\section{MATERIALS AND METHODS}

\section{Display systems}

The medical display system used in this study was a Siemens Model SMM-21140P (Siemens, Erlangen, Germany), having a maximum resolution of

${ }^{*}$ Corresponding author: francis.verdun@chur.ch
$1280 \times 1600$ pixels (i.e. 2 megapixels). According to the manufacturer, its highest luminance was 350 and $800 \mathrm{~cd} \mathrm{~m}^{-2}$ when using a full screen (i.e. $30 \times 40 \mathrm{~cm}^{2}$ ) and a $10 \times 10 \mathrm{~cm}^{2}$ display area, respectively. Its pixel size was equal to $234 \times 250 \mu \mathrm{m}^{2}$. The raster frequency used was systematically set equal to $75 \mathrm{~Hz}$. This display system was connected to a DOME model Md2/PCI graphic board (DOME imaging systems Inc., Waltham, MA), using a 10 to 8 bit $\mathrm{DAC}$ with a video memory of $4 \mathrm{MB}$. This adaptor has an attached photometer of the same mark that served to set up the LUT response of the screen ${ }^{(5)}$.

The desktop display system used was a Philips Model 107 S (Royal Philips Electronics, The Netherlands) using a resolution of $1024 \times 768$ pixels over the $32.5 \times 24.5 \mathrm{~cm}^{2}$ screen surface leading to a pixel size of $317 \times 319 \mu \mathrm{m}^{2}$. There was no technical data available concerning its luminance; however, its highest measured luminance in the $10 \times 10 \mathrm{~cm}^{2}$ geometry was equal to $140 \mathrm{~cd} \mathrm{~m}^{-2}$. The raster frequency used was systematically set equal to $70 \mathrm{~Hz}$. This screen was connected to a standard Trident video accelerator 3D graphic board with a video memory of $8 \mathrm{MB}$. This system was a 32 bit colour display.

\section{Photometer}

The photometer used to perform all luminance measurements was a Gossen Model MAVOMONITOR (GOSSEN Foto und Lichtmesstechnik $\mathrm{GmbH}$, Nürnberg, Germany) using a measurement surface of $6.5 \times 6.5 \mathrm{~mm}^{2}$ and an aperture diameter of $18.5 \mathrm{~mm}$ by means of optical lenses. This device provides a measurement range of $0.01-19,999 \mathrm{~cd} \mathrm{~m}^{-2}$, split into four sub-ranges having a relative uncertainty of $2.5 \%$.

\section{Camera}

The objective measurements were performed using a PMI-1401 C camera, manufactured by 'Q Imaging' 


\section{PERFORMANCE OF DISPLAY MONITORS}

(Q Imaging, Burnaby, BC, Canada). It provides a digital output on 14 bits (16,384 grey levels) and contains a Kodak CCD (Charged Coupled Device KAF-1401) made of $1317 \times 1035$ pixels (pixel size $6.8 \times 6.8 \mu \mathrm{m}^{2}$ ). The camera was read out with an IEEE 1394 (Firewire) interface.

\section{Test patterns}

The AAPM TG18 (AAPM Task Group 18) has developed the image test patterns used. Image quality assessments performed in the framework of this study follow the procedure proposed by AAPM TG18 ${ }^{(3)}$.

\section{Luminance assessment}

The first test to be performed should verify if a display system conformed to the DICOM 3.14 standard $^{\left({ }^{(}\right)}$(which provides the relationship between the grey levels values of the images and the displayed luminance levels). The objective assessment of this parameter is obtained by measuring the luminance in the middle of the 18 TG18-LN test patterns. With these results, the contrast values normalised to their respective just noticeable difference (JND) $)^{(3)}$ can be calculated. A deviation of 10\% from DICOM standard is allowed for first class screens, while a $20 \%$ deviation is authorised for second class screens.

The test image TG18-CT should be used to assess this parameter in a subjective manner. It is made of small low-contrast targets placed in 16 squares of various grey levels. The detectability of all of these targets is required for first class screens.

\section{Spatial resolution assessment}

The test images TG18-RH and TG18-RV, which provide horizontal and vertical impulse lines, respectively, at various luminance levels must be taken with the CCD camera to calculate the presampled modulation transfer function (MTF) of monitors, following the AAPM procedure ${ }^{(3)}$. Once the MTF is obtained, one should verify that its value at the Nyquist frequency is $>25 \%$ for a first class category and for second category monitors this value must be $>35 \%$.

The test image TG18-CX should be used to assess this parameter in a subjective manner. It is made of many CX patterns spread all over the entire image at four luminance levels: maximum luminance level, 75, 50 and 25\%. A 12-grade reference scale (from -2 and -1 for the defocused spot and $0-9$ for perfect to twice the spot size) is provided to assess the spatial resolution of the monitor. The CX elements should be scored between 0 and 4 for a first class display and between 0 and 6 for second class displays.

\section{Image noise}

The noise power spectra (NPS) of monitors can be assessed again with the CCD camera at three luminance levels with the three test images TG18NS that contain large homogeneous areas. There are still no reference values for this parameter.

The test image TG18-AFC can be used to assess this parameter in a subjective manner. It is made of many small boxes containing low-contrast features in a random location; these boxes are placed in four quadrants depending on the contrast and size of the feature. One must detect all the small features of at least three quadrants for a first class display and in two quadrants for a second class display.

\section{RESULTS AND DISCUSSION}

\section{Luminance assessment}

The response luminance curves obtained with the two monitors used in this study are shown in Figure 1. The contrast values normalised to their respective JND are presented in Figure 2. It can be observed that the radiological monitor is within the $10 \%$ tolerance of the ideal curve (i.e. first class category monitor), whereas the desktop monitor diverges from this ideal curve in the low luminance range, and it can be noticed that the desktop monitor is out of the $10 \%$ tolerance range in the low luminance, which is applied to second class radiological monitors.

In visual examination all low-contrast targets could be observed with the diagnostic monitor, while many targets could not be detected in the low and high luminance range with the desktop monitor. This result agrees quite nicely with the results of the objective assessment.

\section{Spatial resolution assessment}

As shown in Figure 3, regardless of the luminance level, the diagnostic monitor offers a pre-sampled MTF, which is systematically $>50 \%$ at its Nyquist frequency (measured equal to $2.14 \mathrm{~mm}^{-1}$ ), thus this monitor can be placed in the first class category.

Figure 4 presents the results obtained with the desktop monitor where a loss of spatial resolution appears with the increase in luminance. Its presampled MTF, at its Nyquist frequency (measured equal to $1.67 \mathrm{~mm}^{-1}$ ), is $<25 \%$ in the medium and high luminance conditions. This makes it a nonacceptable monitor. The degradation of the resolution with the increase of brightness is due to the type of phosphor used, since the diffusion of the light on the surface increases with brightness.

The subjective assessment has given a score of 0 to the radiological monitor, while the desktop monitor obtained a score of 4 in the high luminance range, 


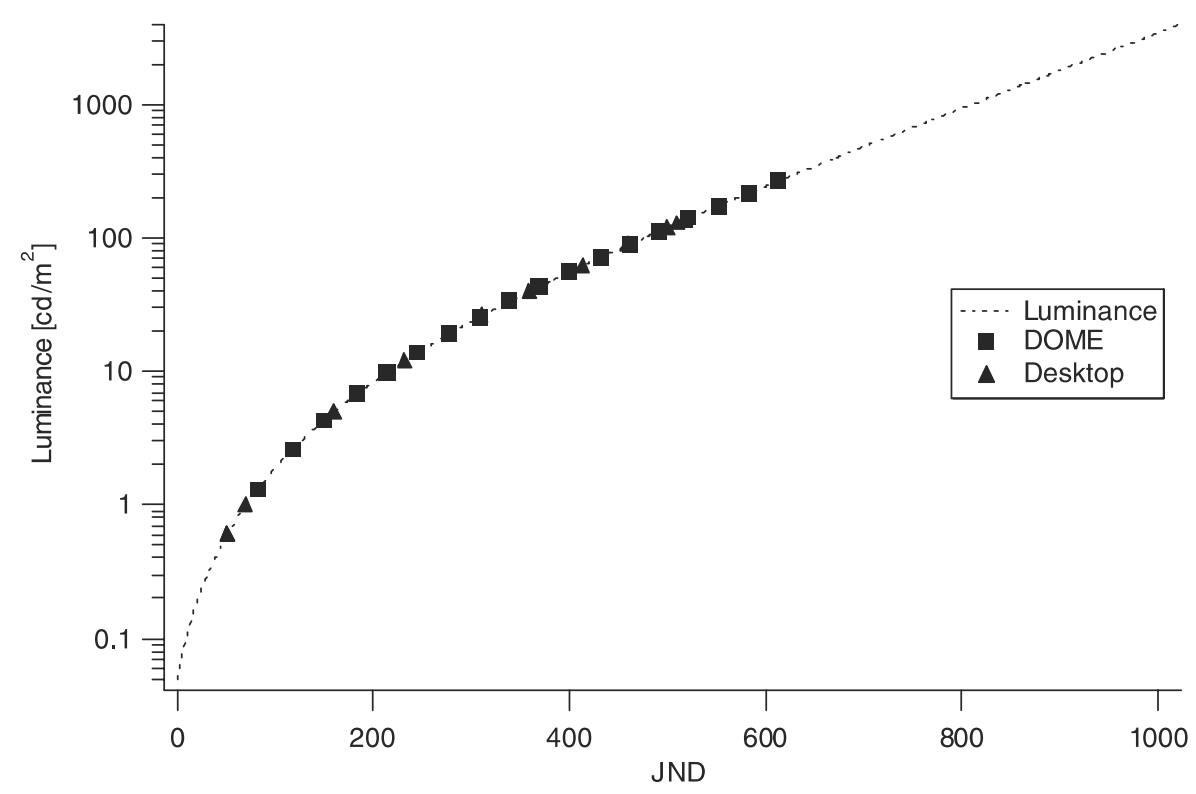

Figure 1. Luminance response corresponding to DICOM 3.14 superposed with our measures.

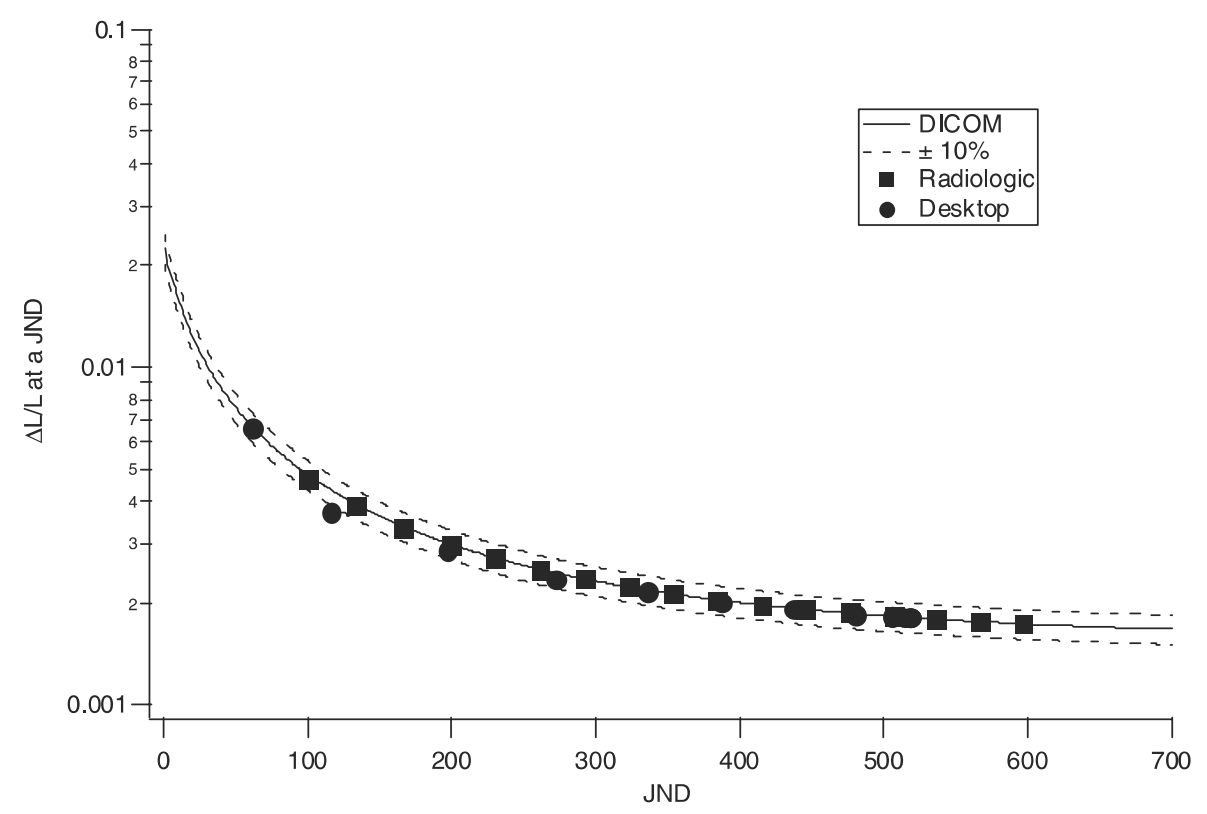

Figure 2. Contrast response from 18 computed patterns for both systems associated with DICOM response.

3 in the medium luminance range and 2 in the low luminance range, confirming the objective results.

\section{Image noise}

Figures 5 and 6 present the NPS obtained at three luminance levels for the radiological and desktop monitors, respectively. The results show that for both monitors normalised noise increases with the luminance level. Moreover, the noise level of the radiological monitor is 10 times lower than that of the desktop one. While there is still no threshold defined by the AAPM standard, it is recommended to have a noise level no 


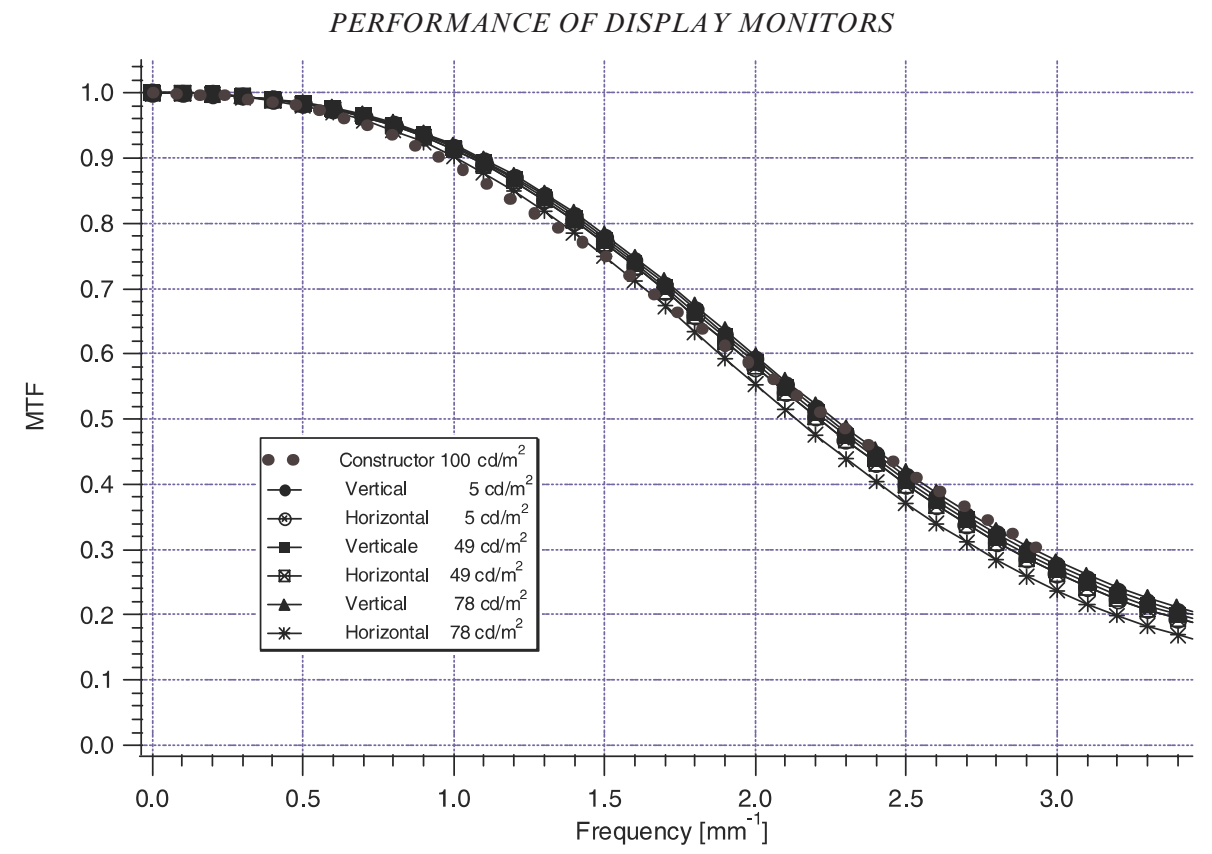

Figure 3. Pre-sampled MTF for three luminance levels of Siemens SMM-21140P calculated by the constructor.

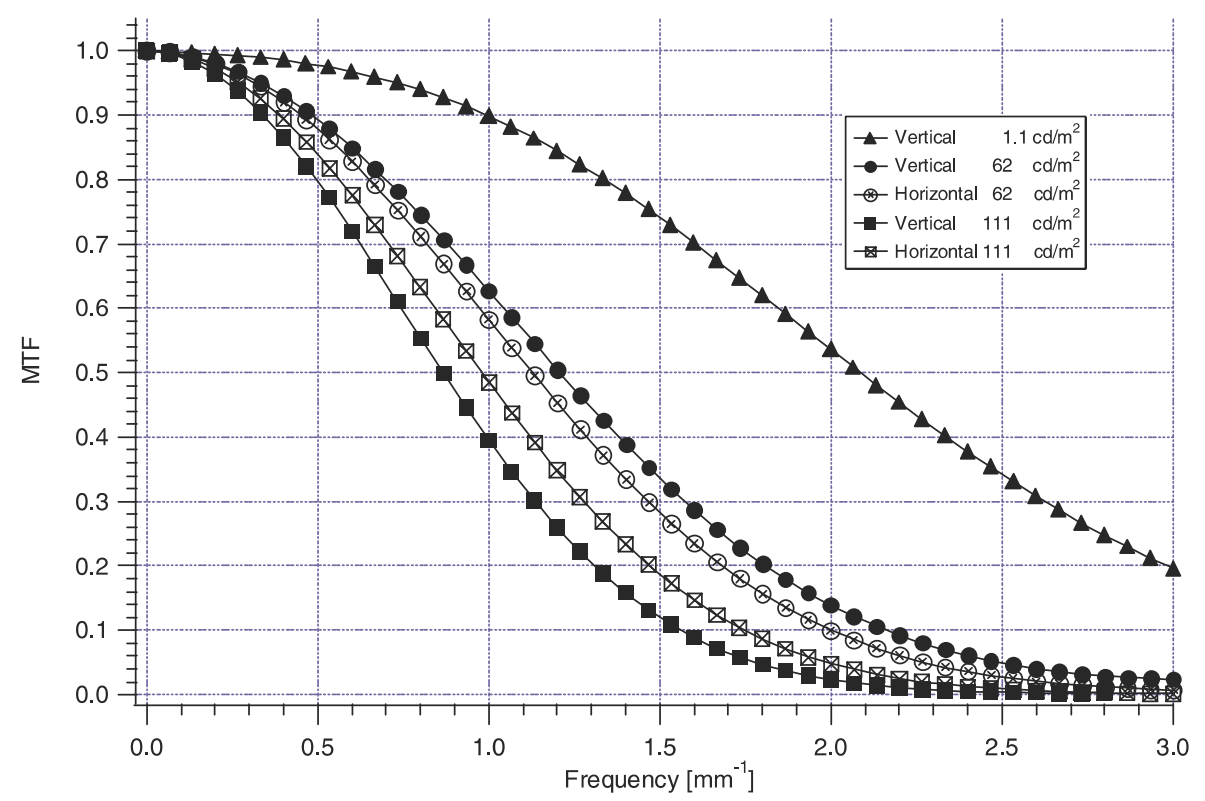

Figure 4. Pre-sampled MTF of the PHILIPS display screen.

higher than the one of a conventional film system.

In both cases peaks corresponding to the raster frequency and associated with the pixel size can be observed (i.e. $4.7 \mathrm{~mm}^{-1}$ corresponding to a pixel size of $\sim 210 \mu \mathrm{m}$ and $3 \mathrm{~mm}^{-1}$ corresponding to a pixel size of $300 \mu \mathrm{m}$ ).

The results of the visual test have ranked the radiological screen as a first class monitor whereas the desktop monitor has been ranked as a second class monitor. 


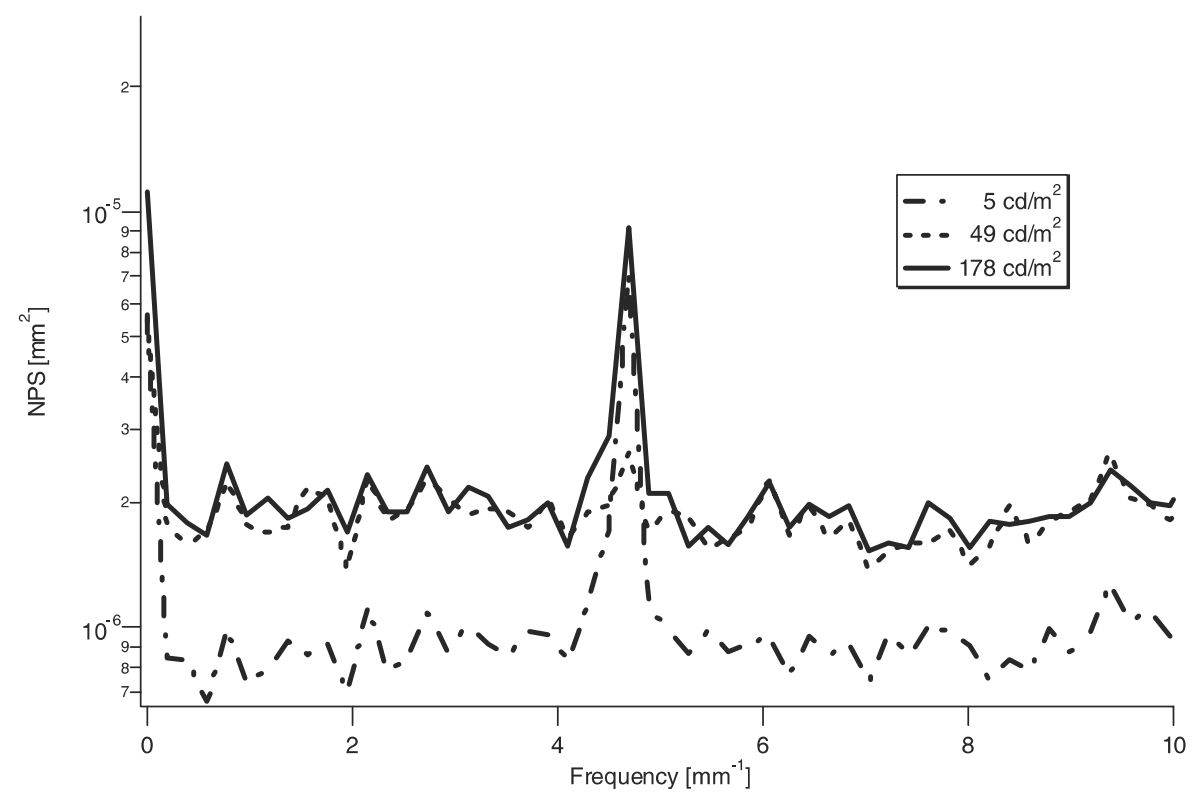

Figure 5. Siemens Weiner spectra for three levels of brightness.

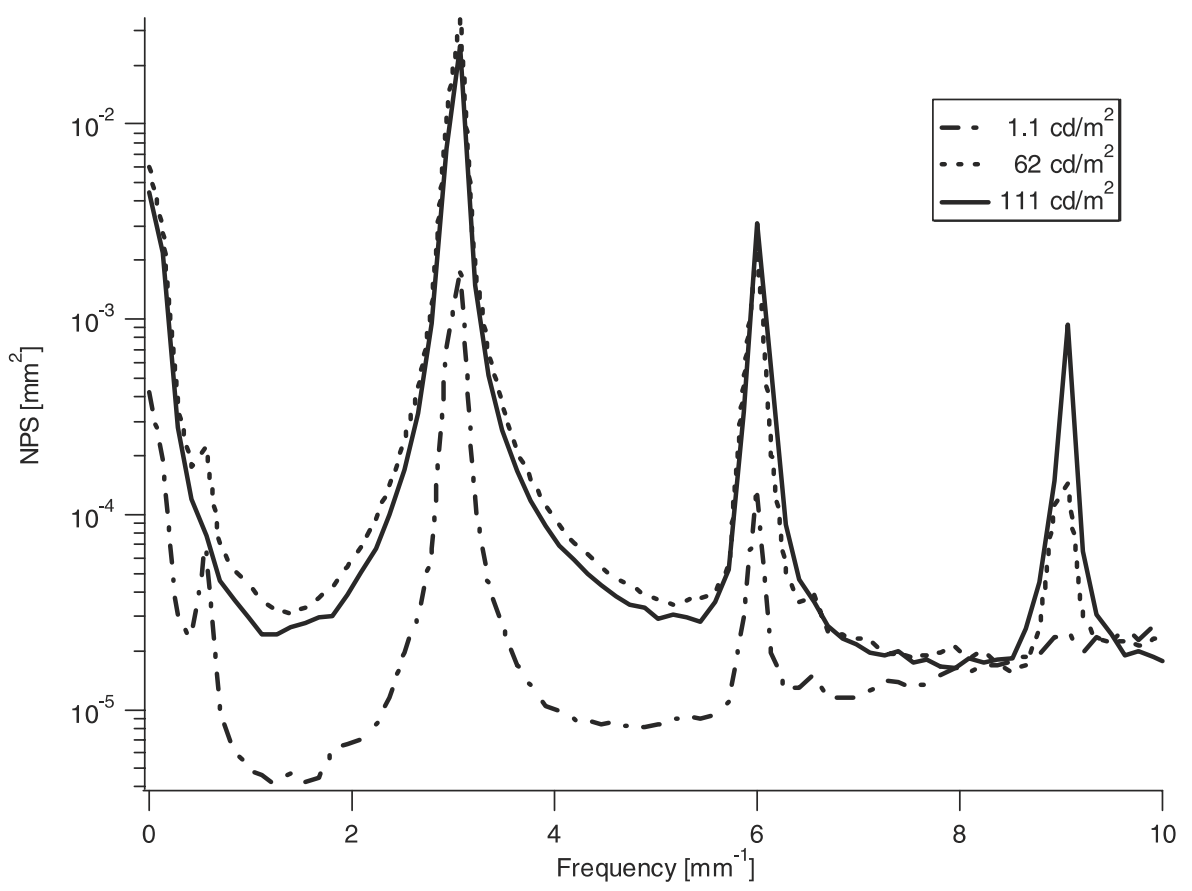

Figure 6. Philips Weiner spectra for three levels of brightness.

\section{CONCLUSIONS}

Several test images proposed by TG18 of the AAPM were tested and provided both subjective and objective results concerning the assessment of two display monitors. These tests are fairly easy to perform and should be integrated into acceptance testing protocols of monitors in radiology 


\section{PERFORMANCE OF DISPLAY MONITORS}

departments. The expected result was confirmed; according to the DICOM 3.14 standard, the desktop display is not suitable to read diagnostic images. A correlation was systematically obtained between the objective and subjective assessments of the monitors. Thus, acceptance tests of monitors do not necessarily require the use of a high-quality level CCD camera. Finally, the weak link, which remains after having accepted a monitor for diagnostic purposes, is its placement. AAPM test images can also be used to demonstrate the importance of ambient light on perceived image quality.

\section{ACKNOWLEDGEMENT}

The authors gratefully acknowledge the financial support of the Swiss Federal Office for Education and Science (grant no 99.0739-Fifth European Research programme no. FIGMCT 2000 00036).

\section{REFERENCES}

1. American College of Radiology. Mammography Quality Control for Medical Physicists (Reston, Va: ACR) (1992).

2. van Voudenberg, S., Thijssen, M. and Young, K. European guidelines for quality assurance in mammography screening. Report EUR 14821, 3rd edition (Luxembourg: CEC) (1999).

3. American Association of Physicists in Medicine Task Group 18. Assessment of display performance for medical imaging systems. Pre-print Draft (version 10.0), August 26 (2004).

4. Radiological Society of North America. Advancesindigital radiography categorical course in diagnostic radiology physics. RSNA Syllabus (Oak Brook, IL: RSNA) (2003).

5. Blume, H. R., Ho, A. M. K., Stevens, F. and Steven, P. M. Practical aspects of grayscale calibration of display systems. In: PACS and Integrated Medical Information Systems: Design and Evaluation. Proc. SPIE 4323, 28-41 (2001).

6. Digital Imaging and Communications in Medicine. Grayscale Standard Display Function DICOM 3 Part 14. National Electrical Manufacturers Association. 